EPJ Web of Conferences 47, 02002 (2013)

DOI: $10.1051 /$ epjconf/20134702002

(C) Owned by the authors, published by EDP Sciences, 2013

\title{
High accuracy \& long timescale light curves
}

\author{
D. Mislis ${ }^{\mathrm{a}}$ and S. Hodgkin
}

\author{
Institute of Astronomy, Madingley Road, Cambridge CB3 OHA, UK
}

\begin{abstract}
We present a theoretical analysis of the optical light curves (LCs) for short-period high-mass transiting extrasolar planet systems. Our method considers the primary transit, the secondary eclipse, and the overall phase shape of the LC between the occultations. Phase variations arise from (i) reflected and thermally emitted light by the planet, (ii) the ellipsoidal shape of the star due to the gravitational pull of the planet, and (iii) the Doppler shift of the stellar light as the star orbits the center of mass of the system. Our full model of the out-of-eclipse variations contains information about the planetary mass, orbital eccentricity, the orientation of periastron and the planet's albedo. For a range of hypothetical systems we demonstrate that the ellipsoidal variations (ii.) can be large enough to be distinguished from the remaining components and that this effect can be used to constrain the planet's mass. As an example we presend KOI-13b (candidate exoplanet system) included in the September 2011 Kepler data release. The Kepler light curve shows both primary and secondary eclipses, as well as significant out-of-eclipse light curve variations. We model the relative contributions from (i) thermal emission from the companion, (ii) planetary reflected light, (iii) doppler beaming, and (iv) ellipsoidal variations in the host-star arising from the tidal distortion of the host star by its companion. Our analysis, based on the light curve alone, enables us to constrain the mass of the KOI-13.01 companion to be $M_{\mathrm{C}}=8.3 \pm 1.25 M_{\mathrm{J}}$ and thus demonstrates that the transiting companion is a planet. The teqnique is useful for current and future space missions such as Kepler and PLATO.
\end{abstract}

\section{INTRODUCTION}

Two observational methods have dominated the study of extrasolar planets so far: radial velocity (RV) measurements and transit light curve (LC) analyses. Both have advantages and disadvantages. While RV determinations provide estimates of the planetary mass $\left(M_{\mathrm{p}}\right)$, the eccentricity (e) and the semi-major axis (a), they do not constrain the inclination (i) of the orbital plane with respect to the observer, thus only lower limits to Mp can be determined. The transit method, on the other hand, provides information on $\mathrm{i}$, the ratio of the planetary to the stellar radius ( $\left.R_{\mathrm{p}} / R_{\mathrm{S}}\right)$, and the duration of the transit (D). So far, only a combination of both strategies yields a full set of orbital and physical parameters for extrasolar planets.

High-accuracy photometry has already been used for a number of systems to show that the planetary thermal emission, as well as the reflection of the stellar light from the planet, are detectable. In particular, Welsh et al. (2010) report the discovery of ellipsoidal variations in the Kepler LC of HAT-P-7. This is an effect more commonly known from close stellar systems, where phase-dependent light variation arises from the gravitationally distorted stars. In HAT-P-7, the planet is close enough and massive enough to induce the same effect.

\section{MODEL}

The main equation we are using in order to model the out-of-transit light of the LC is

$$
f_{3}(z)=\frac{f_{\star}+f_{\text {ref }}+f_{\text {th, } \mathrm{d}}+f_{\text {th, }}+f_{\text {ell }}+f_{\text {dop }}}{f_{\star}} .
$$

\footnotetext{
ae-mail: misldim@ast.cam.ac.uk
}

This is an Open Access article distributed under the terms of the Creative Commons Attribution License 2.0, which permits unrestricted use, distribution, and reproduction in any medium, provided the original work is properly cited. 


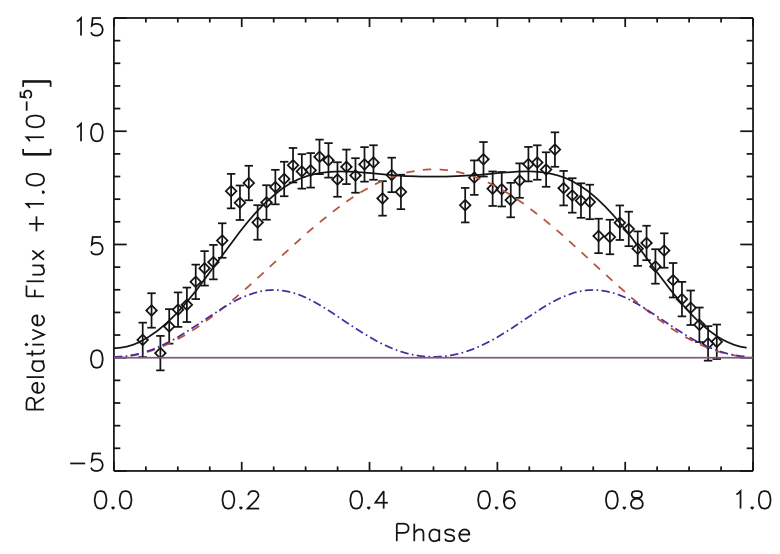

Figure 1. HAT-P-7b data from Kepler. Black solid line is the combine model, red dashed line is the reflected light only, and blue dash-dotted line is the ellipsoidal variations model only.

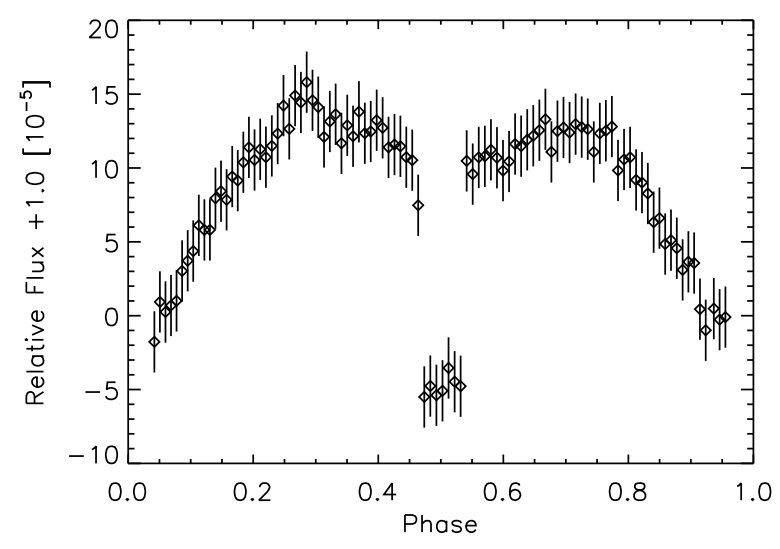

Figure 2. KOI-13b (Kepler data). The primary transit has been removed. After that, variations and the secondary eclipse appears.

where $f_{3}(z)$ is the total light we measure, $f_{\star}$ is the light from the star, $f_{\text {ref }}$ is the reflected light of the planet, $f_{\mathrm{th}, \mathrm{d}}$ is the thermal emission of the planet from the day-side hemisphere, $f_{\mathrm{th}, \mathrm{n}}$ is the thermal emission from the night-side of the planet, $f_{\text {ell }}$ is the ellipsoidal variations and $f_{\text {dop }}$ is the Doppler Beaming effect. For more details of each component and for the analytical equations see Mislis et al. (2012).

We apply first our model in a known system (HAT-P-7b) uing Kepler data. Figure 1 shows the data and the best model fit. The red line in the figure represent the reflected light only model. The reflected light model is not good enough to explain the data, thus we need to include all the additional components.

\section{THE CASE OF KOI-13B}

Using the same analysis as above, and Kepler data, we fit our model in KOI-13b, a Kepler planet candidate. Figure 2 shows the Kepler data, without the primary transit. The depth in phase 0.5 is the secondary eclipse of the planet. KOI-13b is the best example until today, for ellipsoidal variations and Doppler Beaming effect. The reflected only model fails completely to discribe the data. Because both 


\section{Hot Planets and Cool Stars}

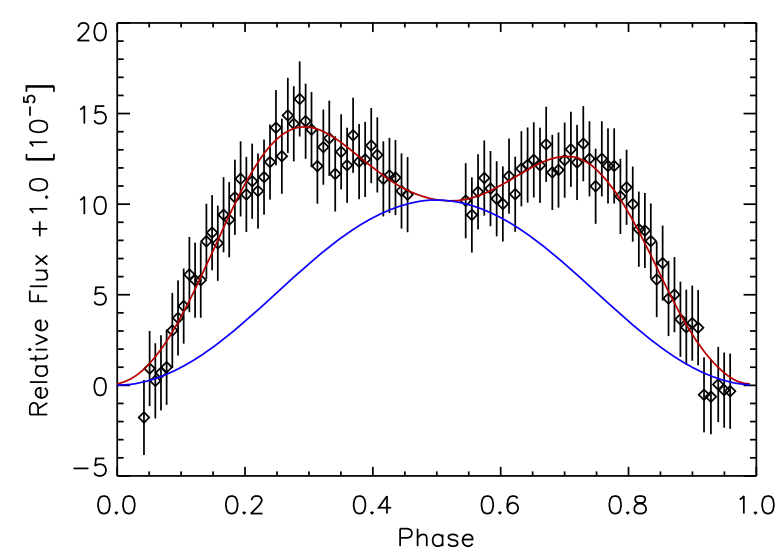

Figure 3. KOI-13b (Kepler data). Red solid line is the combine model, and the blue solid line is the reflected light only model.

ellipsoidal variations and Doppler Beaming components are function of the planetary mass, we can estimate the mass of the planet only from the high accuracy Kepler LC, without any Radial Velocity measurements. Figure 3 shows KOI-13b LC and the best model fit. The planetary mass of the candidate is $M_{\mathrm{C}}=8.3 \pm 1.25 M_{\mathrm{J}}$ (Mislis \& Hodgkin 2012). Mazeh et al. (2011) found a mass of $6 \pm 3 M_{\mathrm{J}}$ and Shporer et al. (2011) found $M_{\mathrm{C}} \sim 9.2 \pm 1.1 M_{\mathrm{J}}$.

\section{CONCLUSIONS}

The mathematical tools can be used for a complete parametrization of transiting exoplanet systems on the basis of high-accuracy LCs only. In our model, for a specific range of systems, RV measurements are not necessary to constrain the mass of the planet, orbital eccentricity, the orientation of periastron, and the geometric albedo of the planet. In order for this method to be fully applicable, the planet must be massive enough and orbit its host star close enough as to distort the stellar structure significantly.

With the current Kepler and potential future missions such as PLATO, we are able to measure the mass of the hot Jupiters with orbital periods less than 10 days. Furthermore, for host stars with spectral types earlier than FV, thermal emission component is detectable in the LC (assuming black bodies). This will affect the accuracy and even the detection of ellipsoidal variation. As we show, the Kepler mission provides an accuracy suitable enough for our procedure to be applied for the characterization of extrasolar planets.

Using ellipsoidal variations we calculate the planetary mass from the photometric LC itself, without any RV measurments. Our technique will benefit from future space missions such as PLATO and the James Webb Space Telescope (Deming et al. 2009).

\section{References}

[1] Deleuil M., ASPC, 119, 259, (1997)

[2] Mazeh T., A\&A, 59M, 521, (2010)

[3] Mislis D., A\&A, 4M, 538A, (2012)

[4] Mislis D., MNRAS, 1512M, 422, (2012)

[5] Shporer A., AJ, 195S, 142, (2011)

[6] Welsh F. ApJ, L145, 713, (2010) 